\title{
Does molecular electronics compute?
}

\author{
The field of molecular electronics originally set out to build computers, but silicon-based technology is \\ unlikely to be replaced anytime soon. Nevertheless, the field has developed into a highly interdisciplinary \\ endeavour, which could have a variety of ramifications that extend beyond computing.
}

Molecular electronics can be traced back to the early $1970 \mathrm{~s}^{1}$, but it is only in the past decade that important experimental and theoretical hurdles have finally been overcome. Experiments with single-molecule junctions, for example, have become more robust, reliable and reproducible ${ }^{2}$. At the same time, theoretical methods based on Green's function theory have been developed and have allowed researchers to investigate the fundamental properties of single molecules under nonequilibrium conditions ${ }^{3}$. These advances have led to the discovery of a host of novel effects that have only recently been systematically investigated. Concurrently, a variety of potential practical applications, which extend beyond the electronic devices predicted in the early days of the field, have also been proposed ${ }^{4}$.

In this issue of Nature Nanotechnology we provide an overview of the current state of molecular electronics. To begin, we asked 12 leading researchers in the field to discuss their motivations and share their view of the future of molecular electronics ${ }^{5}$. The list of researchers is inevitably incomplete (and we apologize to those that were left out), but it is clear from these contributions that there is no single way forward for the field: it attracts researchers from a variety of backgrounds and is beginning to branch out in different directions reflecting its interdisciplinary realm.

One of the most important works in the history of molecular electronics appeared in the journal Chemical Physics Letters in 19746. Arieh Aviram and Mark Ratner, who at the time were at IBM's Thomas J. Watson Research Center and New York University, respectively, suggested that a single molecule could function as a rectifier ${ }^{6}$. In the first Commentary in this issue, Mark Ratner, who is now at Northwestern University, reflects on the various phases the field has gone through since that time and where it should go next ${ }^{7}$. In particular, he urges researchers to move beyond a merely qualitative description of electron transport through a molecule and to develop theoretical models that can fully describe

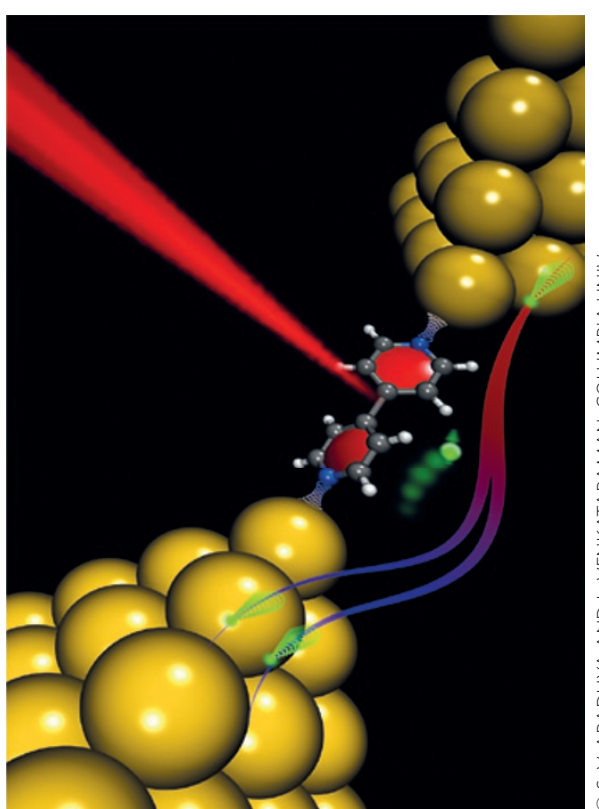

To think of molecules as only being a substitute for silicon is limiting. As Mario Ruben of the Karlsruhe Institute of Technology succinctly put it at the recent conference 'Future Directions in Molecular Electronics' held in Leiden: "to do Boolean logic with molecules is to do violence against them". Molecules can do so much more and we are only beginning to understand the possibilities. Intriguing spin quantum effects at room temperature have, for example, been observed in single molecules, attracting a lot of interest to the relatively new field of molecular spintronics, which could have exciting implications for the development of practical quantum computing 9 . To appreciate how far the molecular electronics field has come and where it is going, the Review by Sriharsha V. Aradhya and Latha Venkataraman of Columbia University is a good starting point ${ }^{10}$. It discusses recent advances in the

the quantum effects that are typical at the molecular level.

The electronics industry will stick with complementary metal-oxidesemiconductor (CMOS) technology for as long as it is economically viable and it is unlikely it will be phased out for at least another decade. There is, however, an inevitable limit to the miniaturization of transistors, and molecules can, in principle, provide a precise spatial control that is currently impossible with topdown fabrication methods. In the second Commentary, Emanuel Lörtscher of IBM Zurich explains what would need to be done for a viable molecular electronics technology to emerge $e^{8}$. It is imperative to decrease the variability that is observed in single-molecule junction experiments - a statistical approach, although very informative for fundamental studies, would not be suitable for applications. Moving away from gold electrodes may be a promising way forward. Lörtscher notes that any switch from CMOS technology to molecular components would at first be gradual, and molecules with specific functions would initially just complement existing technologies. characterization at the single-molecule level of, among others, mechanical, thermal, spin and quantum properties.

The original vision for molecular electronics, for now, remains unfulfilled. The field has, however, become a playground for fundamental studies. Chemists, for example, who have always studied the structureproperty relationship of molecules, now have the possibility to characterize their systems at the most fundamental level possible. Understanding the behaviour of single molecules can change the way we approach practical problems in, for instance, solar-energy harvesting, thermoelectrics, catalysis and sensing. This focus issue will hopefully provide a glimpse of the opportunities that exist.

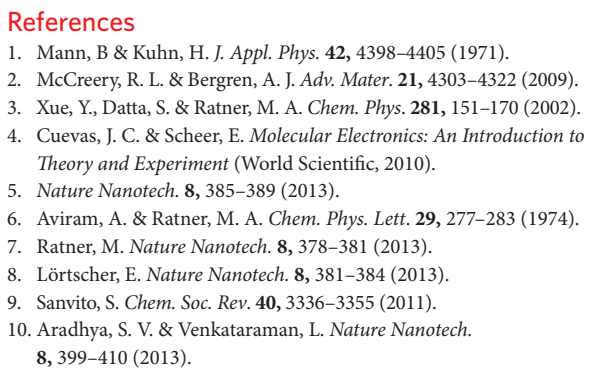

